La FASP : dix ans après...

\title{
La FASP comme outil pédagogique en anglais spécialisé pour étudiants de psychologie
}

Jacqueline Hamrit

\section{OpenEdition \\ Journals}

Édition électronique

URL : http://journals.openedition.org/ilcea/498

DOI : 10.4000/ilcea.498

ISSN : 2101-0609

Éditeur

UGA Éditions/Université Grenoble Alpes

Édition imprimée

ISBN : 978-2-84310-180-9

ISSN : $1639-6073$

Référence électronique

Jacqueline Hamrit, «La FASP comme outil pédagogique en anglais spécialisé pour étudiants de psychologie », ILCEA [En ligne], 12 | 2010, mis en ligne le 29 septembre 2010, consulté le 30 avril 2019. URL : http://journals.openedition.org/ilcea/498 ; DOI : 10.4000/ilcea.498

Ce document a été généré automatiquement le 30 avril 2019

(C) ILCEA 


\title{
La FASP comme outil pédagogique en anglais spécialisé pour étudiants de psychologie
}

\author{
Jacqueline Hamrit
}

\section{Introduction : l'enjeu de la fiction en psychologie, en formation continue et en formation initiale}

Dans la perspective d'une mise en place d'un enseignement de textes de fiction en formation initiale en anglais d'étudiants en psychologie, cet article présente l'analyse d'un ouvrage qui répond à certains critères principaux de la FASP. Il s'agit du best-seller de Daniel Keyes, à savoir Flowers for Algernon. Mon intérêt pour la FASP - fiction à substrat professionnel - est relativement récent. Il a été, en effet, suscité, comme de nombreuses activités de recherche, par l'enseignement, notamment dans le cadre de la mise en place d'un diplôme universitaire. Ce diplôme, offert en formation continue à Lille 3, proposait les outils d'une réflexion épistémologique et philosophique sur la relation clinique à partir de la phénoménologie, de la psychanalyse et de la littérature et s'adressait à un public de professionnels de la psychiatrie et de la psychologie. Titulaire d'une thèse de doctorat en littérature américaine, je suis intervenue dans le troisième module du programme, la partie littéraire. J'ai choisi de présenter des œuvres de Flaubert, Fitzgerald, Melville et Nabokov. Mon objectif était d'aller au-delà de l'entreprise commune qui consiste à faire entrer en résonance la psychanalyse et la littérature, et de proposer à des spécialistes de la psychologie proprement dite une réflexion sur les pratiques cliniques en les abordant sous leur aspect le plus insaisissable : la subjectivité. Dans cette perspective, j'ai entrepris de montrer comment la littérature pouvait être un outil utile à l'enseignement de la psychologie et comment le recours à la littérature permettait d'envisager le discours spécialisé non seulement comme un ensemble de savoirs formels, terminologiques ou autres, mais comme un contenu ayant du sens. Je considère, en accord avec Michel Petit (2004), qu'un domaine de spécialité - comme la psychologie, par exemple -, se présente comme un savoir qui peut être appréhendé par 
différents types de discours - un discours que Petit nomme " primaire » comme, dit-il, on parle de « sources primaires » en civilisation : on peut ainsi retenir l'article de recherche, l'étude de cas, etc., et aussi le discours littéraire. D'où mon intérêt pour la FASP, qui, par sa dénomination, souligne la dimension fictionnelle éventuelle d'un discours spécialisé.

L'expérience en question concernait la formation continue (l'enseignement était effectué en français puisque le but pédagogique n'était pas l'apprentissage de l'anglais). La lecture de l'article fondateur de Michel Petit de 1999 sur la FASP, publié dans la revue du GERAS, Asp, m'incita à concevoir un nouveau projet pédagogique, à savoir la mise en place d'un enseignement de textes fictionnels dans le cadre de la formation initiale destinée aux étudiants en psychologie. Le support que je compte utiliser et que je me propose d'étudier ici est le best-seller de l'américain Daniel Keyes intitulé Flowers for Algernon. Cet ouvrage répond, me semble-t-il, à certains critères principaux de la FASP, telle qu'elle est définie par Michel Petit. Il écrit, en effet : «peut-être considéré comme ressortissant de la FASP tout texte de fiction commerciale à grand succès (i) relevant généralement du thriller spécialisé (juridique, médical, technologique, etc.), (ii) utilisant un milieu professionnel particulier non seulement comme cadre général de l'histoire mais aussi et surtout comme l'une des sources principales des ressorts de l'intrigue, (iii) exprimé dans une langue reproduisant les pratiques langagières (lexicales et discursives) de ce milieu, et (iv) généralement écrit par un auteur dont l'appartenance ou les liens avec ce milieu sont explicitement revendiqués. » (Petit, 1996, p. 5)

2 Ainsi, dans le cas qui nous intéresse, l'auteur - né à Brooklyn (New York) en 1927 - a non seulement poursuivi des études en littérature anglaise et américaine qu'il a ensuite enseignée au Brooklyn College puis à l'Université de l'Ohio, mais a également obtenu un diplôme en psychologie. L'ouvrage fut vendu à plus de cinq millions d'exemplaires, a été traduit en vingt-sept langues et publié dans trente pays. Il a obtenu le prix Nebula du meilleur roman en 1966, après que la nouvelle dont est issu le roman eut obtenu le prix Hugo de la meilleure nouvelle en 1960ํ․ L'histoire fut adaptée à la télévision en 1961 dans The Two Worlds of Charlie Gordon avec Cliff Robertson comme acteur principal, puis au cinéma en 1968 dans un film intitulé Charly avec encore Cliff Robertson qui remporta un Oscar pour sa performance, puis au théâtre en 1969, à la radio en 1991 pour BBC Radio 4 et enfin à la télévision française en 2006. Toutes ces adaptations sont, bien sûr, un bonus pédagogique puisqu'elles ouvrent des perspectives d'exploitation pour les compétences orales. Le livre appartient au domaine de la science-fiction. Il traite cependant de la recherche et des expériences en psychologie. Il raconte, en effet, l'histoire d'un attardé mental, Charlie Gordon, qui subit une opération de psychochirurgie ${ }^{2}$, censé le rendre intelligent. L'opération réussit dans un premier temps : de 68, son QI grimpe à 185. Il maîtrise plusieurs langues étrangères, fait de la recherche en psychologie, résout des problèmes inédits en psychologie expérimentale. Mais, un jour, les facultés supérieures de la souris de laboratoire Algernon, qui avait subi auparavant la même opération que lui déclinent. Charlie doit alors faire face au drame d'un homme qui, en pleine conscience, se sent retourner à l'état de bête.

3 Il semble donc que l'ouvrage de Keyes réponde à de nombreux critères génériques de la FASP, ne serait-ce que par les caractéristiques personnelles de son auteur (psychologueécrivain), son succès commercial et son cadre professionnel. 


\section{Un substrat professionnel et non un simple adstrat}

4 J'aimerais maintenant développer deux plans d'analyse. Le premier portera sur la présence du substrat professionnel. La deuxième partie de l'analyse de l'ouvrage traitera de la façon dont le substrat professionnel est bien « substrat » et non « adstrat » puisqu'il détermine l'intrigue.

5 Petit écrit, je le rappelle, qu'une œuvre relevant de la FASP utilise «un milieu professionnel particulier non seulement comme cadre général de l'histoire mais aussi et surtout comme l'une des sources principales des ressorts de l'intrigue.» (Petit, 1999, p. 5) Je souhaiterais donc développer les deux parties de cette définition du substrat professionnel et montrer d'abord que le cadre appartient bien à celui de la psychologie en me référant, dans un premier temps, à divers manuels de synthèse de la psychologie, recommandés aux étudiants de psychologie de mon UFR, et en y recensant les thèmes généraux, puis, dans un deuxième temps, en essayant de retrouver ces mêmes thèmes dans l'ouvrage de Keyes. Ainsi, la lecture des tables des matières de l'ouvrage d'Alain Lieury intitulé Psychologie cognitive (2004), ainsi que de l'ouvrage de Jo Godefroid, Psychologie (2008) fait apparaitre de nombreuses convergences. Le livre de Lieury comporte huit chapitres, portant respectivement sur l'histoire de la psychologie, la perception, l'apprentissage, la mémoire, le langage, l'intelligence, la motivation, les émotions et la personnalité. Celui de Jo Godefroid, plus fouillé, est composé de sept parties et de vingt chapitres. Il envisage la psychologie en tant que science, et plus particulièrement en tant que science humaine. Il traite, entre autres, des questions de biologie, perception, mémoire, pensée, langage, conscience, vie affective, motivation, émotions, ainsi que celles de l'intelligence, de la personnalité, de la psychopathologie et des thérapies. Or, la majorité de ces thèmes apparaissent dans le roman de Keyes. Il est ainsi question d'intelligence, de la pensée et de la mémoire, du langage, du conscient et de l'inconscient, de la motivation. Des émotions - comme la colère, la peur, la honte, par exemple -, sont analysées. Y sont également traités les thèmes propres à la psychologie, tels que la personnalité et les processus interpersonnels, le quotient intellectuel et émotionnel, les tests, la science expérimentale, l'apprentissage, les psychothérapies ainsi que le cas particulier du retard mental, de la norme et du handicap. Le livre baigne donc bien dans ce que Petit appelle « une culture professionnelle » qu'il définit ainsi : « Nous entendons ici par culture d'un milieu spécialisé l'ensemble des éléments qui le décrivent en tant que milieu, c'est-à-dire qui constituent le fonds commun de ce milieu : savoirs et savoir-faire partagés, modes de pensée et d'expression, modes d'organisation collective, pratiques, us et coutumes, objets, outils, etc.» (Petit, 1999, p. 10) L'ouvrage, de fait, n'inclut pas seulement des thèmes traitant de la psychologie mais il présente également la rédaction d'un article de recherches en psychologie écrit par Charlie alors au summum de ses capacités intellectuelles. Il raconte un congrès de chercheurs en psychologie à Chicago. Il décrit les passations de tests expérimentaux, notamment celui de Rorschach.

6 Pourtant, l'ouvrage offre plus qu'un simple cadre référentiel lié à la psychologie. Le substrat professionnel est bien substrat et non adstrat. Petit précise bien dans une note de son article publié en 2004 dans Aspects de la fiction à substrat professionnel comment « le fait qu'une enquête policière de fiction se déroule, par exemple, dans le milieu des musiciens professionnels ou des courses hippiques ne peut suffire à permettre de parler de FASP musicale ou hippique. » (Petit, 2004, p. 20) Shaeda Isani, dans le même numéro 
sur la FASP, explicite la différence entre substrat professionnel et adstrat professionnel de la façon suivante : «Dans sa définition des conventions essentielles de la FASP, Petit fait la distinction entre la fiction où la profession joue un rôle central (le substrat professionnel) et la fiction où la profession est largement présente mais pas de façon indispensable et pour laquelle il propose le terme d'adstrat professionnel ${ }^{3}$.» Isani précise dans une note : «Adstrat : terme français emprunté au domaine de la linguistique et qui désigne une langue parlée dans un pays avoisinant le pays de référence, qui, dans le contexte de notre étude, implique la notion de juxtaposition comme étant opposée à celles d'origine ou de fondement $t^{4}$.»

\section{Le substrat professionnel fait partie intégrante du récit}

7 J'aimerais donc montrer comment, au contraire, dans Flowers for Algernon, le substrat professionnel détermine l'intrigue, comment le lien qui unit l'histoire et le cadre professionnel est celui de l'entrelacs et non de la juxtaposition, comment, en d'autres termes, le récit est conditionné par le milieu professionnel. Le récit est d'abord une intrigue structurée par des éléments temporels. Dans l'ouvrage qui nous occupe, l'étude du récit peut s'appréhender sous trois angles différents, à savoir la structure, les thèmes, et la narration proprement dite. Le livre est, en effet, de forme épistolaire : il est composé de dix-sept comptes rendus, écrits par le protagoniste qui raconte sa vie quotidienne avant l'opération et après celle-ci. Les comptes rendus, datés du 3 mars au 21 novembre, couvrent donc une période de neuf mois. L'histoire suit une ligne régulière particulière qui forme d'abord un crescendo qui se manifeste par une évolution entre l'expression pauvre et incorrecte des débuts (quand le protagoniste est à un QI de 68) aux explications riches et complexes lorsqu'il est au sommet de ses capacités intellectuelles. Puis l'histoire raconte le déclin des capacités du protagoniste qui retrouve à la fin de l'histoire l'expression pauvre et incorrecte du début. La structure circulaire, ou plutôt en spirale (Charlie fait preuve à la fin d'une maturation psychologique due au souvenir de son expérience), est enrichie des images et symboles auxquels l'auteur a recours. Ainsi, en exergue, Keyes cite un extrait de La République de Platon qui rappelle comment l'aveuglement peut avoir deux causes : un excès de lumière ou l'inaccoutumance au noir. Il est difficile de voir et donc de savoir quand on sort de la lumière ou quand on y rentre. Il s'agit là d'une image qui rappelle l'expérience de Charlie, qui des ténèbres de la bêtise intellectuelle entre trop violemment dans la lumière aveuglante du savoir et de l'intelligence pour enfin retrouver tragiquement l'obscurité de l'incompréhension. Un autre symbole présent dans le livre est celui du labyrinthe -le labyrinthe que les expérimentateurs utilisent pour tester et comparer les capacités cognitives de la souris Algernon ainsi que celles de Charlie qui doit se confronter au début de son expérience au test du labyrinthe. C'est le labyrinthe perfectionné en trois dimensions que Charlie confectionne pour Algernon lorsqu'il s'enfuit avec elle afin de perfectionner les facultés de celle-ci. C'est le labyrinthe, (maze, en anglais) - Charlie comprend "amazed» qui représente les sinuosités et les tâtonnements de sa nouvelle vie, avec sa perplexité, ses frustrations, ses réussites et ses échecs. C'est enfin la fenêtre qui constitue le cadre des images représentant Charlie enfant que Charlie adulte et opéré voit et se remémore.

8 Or, le cadre référentiel qui entoure l'histoire de Charlie est, comme nous l'avons vu, alimenté par une thématique relevant de la psychologie. Ainsi les objets et les outils qui, selon Petit, appartiennent à la culture d'un milieu spécialisé font partie intégrante de 
l'intrigue. Charlie, en effet, se rend, avant et après l'opération, au laboratoire de psychologie, rencontre des psychologues qui lui font passer des tests d'aptitude. Les tests sont décrits, parfois nommés ou identifiés. De même, certaines approches méthodologiques sont mentionnées. Ainsi, Charlie critique indirectement le béhaviorisme lorsqu'il reproche aux expérimentateurs d'utiliser pour tester Algernon la méthode de la punition, le choc électrique, et de la récompense, la nourriture. L'auteur semble, en revanche, être en faveur de l'approche psychanalytique dont il décrit la méthode de l'association libre laquelle permet à Charlie de comprendre ses souvenirs et ses sentiments.

9 Je prendrai enfin deux autres exemples pour montrer comment la thématique s'intègre au récit. Le premier se situe vers le milieu de l'histoire lorsque Charlie, amoureux de son professeur Alice Kinnian, essaie de faire l'amour avec elle mais n'y parvient pas car il se sent submergé par une peur panique et a l'impression d'être épié. Il écrit :

Quand je la pris par les épaules, elle se raidit et frémit mais je l'attirai à moi... C'est alors que cela se produisit. Cela commença par un bourdonnement sourd dans mes oreilles... un bruit de scie électrique... très loin. Puis une sensation de froid: des picotements dans mes bras et mes jambes, mes doigts engourdis. Soudain, j'eus la

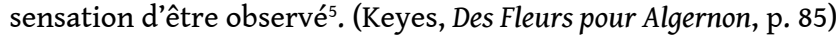

10 L'auteur décrit là les effets physiologiques de la peur, ainsi qu'une hallucination, voire un dédoublement de personnalité qui tend vers une expérience paranoïaque. Il intègre donc les éléments psychologiques à l'intrigue proprement dite puisque le fait que Charlie soit, comme il le dit un peu plus tard, « sexuellement retardé » (Keyes, Des Fleurs pour Algernon, p. 87) [« sexually retarded» (Keyes, Flowers for Algernon, p. 102)] influe sur le récit. En effet, il s'éloigne d'Alice et se lie à une autre femme, Fay, avec qui il réussit à avoir des relations sexuelles. L'autre exemple concerne l'expérience qu'a Charlie de ce qu'on appelle en psychologie le phénomène de l'insight qui correspond à une compréhension soudaine et fulgurante de quelque chose. Vers la fin de l'histoire, Charlie essaie de trouver la solution à la faille inhérente à l'expérience de psychologie qu'il a subie. Il s'écrie alors :

La solution m'est venue, alors que je somnolais. Lumineuse! Tout se raccorde et je vois ce que j'aurais dû savoir depuis le début. Assez dormi. Il faut que je retourne au labo et que je vérifie cela avec les résultats de l'ordinateur. C'est là, finalement, la faille dans l'expérience. Je l'ai trouvée ${ }^{6}$. (Keyes, Des Fleurs pour Algernon, p. 207)

Cette découverte va susciter l'écriture d'une lettre au responsable de l'expérience, le professeur Nemur, et va déterminer la prise de conscience de Charlie de sa future déchéance.

Or, au cœur de l'entrelacs entre substrat professionnel et intrigue se trouve la narration proprement dite. Le protagoniste connaît, en effet, une modification de ses capacités cognitives et émotionnelles, ce qui crée attente chez le lecteur et suspense dans le récit. Les étapes de l'évolution du protagoniste sont mises en relief car elles sont souvent mentionnées au début des comptes rendus. Ainsi, le compte rendu daté du 4 mars commence par la courte phrase : «J'ait passé un teste ojourdui. » (Keyes, Des Fleurs pour Algernon, p. 7) [ "I had a test today. " (Keyes, Flowers for Algernon, p. 1)]; celui du 11 mars par la phrase tout aussi courte : «L'opération ne m'a pas fait mal.» (Keyes, Des Fleurs pour Algernon, p. 17) [" The operashun dint hert. » (Keyes, Flowers for Algernon,p. 13)] et enfin celui du 29 mars par ce qui correspond à un événement et une rupture dans son évolution: «J'ai battu Algernon. » (Keyes, Des Fleurs pour Algernon, p.31) [ «I beet Algernon.» (Keyes, Flowers for Algernon, p.31)] Ces exemples se situent au début du roman lorsque Charlie devient de plus en plus intelligent. Lorsqu'il décline, le suspense est différent. Le lecteur, 
prévenu par les nombreux signes avant-coureurs, attend et s'attend à ce qui va arriver : il envisage le déclin et le voit arriver. Par ailleurs, le lecteur se trouve confronté à un protagoniste-narrateur qui joue sur les écarts d'informations entre narrateur, personnage et lecteur, ce qui crée une forte ironie dramatique. Ainsi, Charlie passe le test de Rorschach deux fois : avant et après l'opération. Avant l'opération, Charlie ne sait pas ce qu'est un test de Rorschach. D'ailleurs, il ne sait pas comment écrire le mot. Mais le narrateur, en faisant comprendre qu'il s'agit de ce test, crée par le recours à l'implicite, une complicité entre le lecteur et lui-même. Il décrit la passation du test de telle sorte que le lecteur est censé deviner alors que le narrateur, quant à lui, feint de ne pas comprendre. L'implicite est également présent dans les allusions à certains regards appuyés et étranges des autres personnages sur Charlie. Ainsi, Keyes écrit : «M. Donner m'a regardé drôlement un bon moment.» (Keyes, Des Fleurs pour Algernon, p.25) [« M . Donner looked at me for a long time funny. " (Keyes, Flowers for Algernon, p.24)] Charlie est supposé ne pas décoder le regard alors que le lecteur est censé pouvoir le faire. Ce jeu entre implicite et explicite, réapparait à la fin du livre quand Charlie reperd son intelligence et ne comprend plus, de ce fait, ce qu'Alice veut dire. Keyes écrit :

Elle procédait par allusions. Elle évitait de parler directement, et elle espérait que je comprendrais ce qu'elle pensait. Et j'écoutais, faisant semblant de comprendre mais au fond de moi, j'avais peur qu'elle ne voie que je n'avais pas du tout saisi son propos? $^{7}$. (Keyes, Des Fleurs pour Algernon, p. 243)

13 Ici, Charlie a l'impression qu'Alice parle de façon implicite - d'où l'effet ironique. La fin de l'histoire correspond aux pertes en tous genres - pertes des capacités cognitives et émotionnelles, pertes des relations sociales, éclatement de l'identité. S'étant fréquemment interrogé sur, dit-il, ce qu'il est et quiil est [" who and what I am »], il écrit à la fin : «Je m'effondre par morceaux. » (Keyes, Des Fleurs pour Algernon, p. 244) [«I'm falling apart. » (Keyes, Flowers for Algernon, p. 300)] Le thème de l'identité - élément central en psychologie - rejoint celui que Ricoeur appelle l'identité narrative où le protagoniste raconte son histoire et se raconte. On nomme, en effet, les récits de patients, des «pathographies.»

\section{Au-delà de l'exploitation pédagogique de la FASP : la dimension éthique}

14 On peut alors se poser la question : peut-on utiliser l'ouvrage de Keyes pour des cours en psychologie et, plus généralement, pourquoi avoir recours à la FASP lors des cours de langue à de futurs psychologues ? Le livre est, évidemment, tout d'abord un support tout à fait légitime pour une exploitation pédagogique normale avec étude lexicale, grammaticale, discursive, entrainement aux compétences de compréhension et de production. Il me semble, cependant, que la FASP - par les émotions qu'elle peut susciter chez le lecteur - permet non seulement de développer chez le psychologue la capacité indispensable à avoir de l'empathie mais également de s'interroger sur la dimension éthique de sa profession. Ricoeur, en parlant de la psychanalyse, explique que le patient qui raconte sa vie cherche à créer un récit avec lequel la vie sera plus supportable et plus intelligible. (Ricoeur, p. 114) Raconter sa vie, c'est donc, pour lui, donner du sens à sa vie, se construire une identité mais aussi maitriser sa souffrance. Or le livre de Keyes donne un exemple probant de ce phénomène. Charlie souffre. Il souffre de la maltraitance et de la cruauté de sa mère qui refusait d'admettre son handicap quand il était enfant. Il souffre 
parce qu'on se moque de son retard mental, qu'on le traite comme un animal. Même les psychologues du laboratoire ne l'utilisent que comme un cobaye et lui accordent que peu d'attention en tant que, pour reprendre son expression, « personne humaine ». Le lecteur éprouve de la compassion pour lui. Dans un article en ligne du nytimes.com daté du 23 octobre 2008, et qui a pour titre "Stories in the Service of Making a Better Doctor" ", il est fait mention du Docteur Panush qui, ayant prescrit à ses internes la lecture de textes littéraires ayant trait à la médecine, a pu vérifier les effets bénéfiques de cette pratique. Ils devenaient, dit-il, de meilleurs médecins. La FASP, enseignée à des étudiants de psychologie, pourra en faire peut-être, également, de meilleurs psychologues.

\section{Conclusion : comment élargir la perspective?}

15 Je souhaiterais enfin, pour conclure, élargir la perspective en me penchant sur les allusions intertextuelles présentes dans le roman de Keyes. Ces allusions ont trait à la question de la science en général et sont associées à la Bible, à la tragédie grecque et à la littérature anglo-saxonne. À plusieurs reprises, des personnages - souvent féminins dénoncent l'aspect transgressif de l'expérience du Professeur Nemur. Voilà ce que dit une infirmière de l'hôpital où Charlie se fait opérer :

Et elle a dit que peut-être ils n'avaient pas le droit de me rendre intelligent parce que si Dieu avait voulu que je sois intelligent, il m'aurait fait naître intelligent. Et il ne faut pas oublier Adam et Eve, et le péché avec l'arbre de la science, et la pomme mangée et la chute'. (Keyes, Des Fleurs pour Algernon, p.19)

Une transgression similaire apparait lorsque Charlie mentionne les péchés de ses ancêtres et s'écrie :

J'aurais voulu pouvoir lui dire que, comme la famille grecque des Atrides, nous payions pour les péchés de nos ancêtres, ou accomplissions un ancien oracle ${ }^{10}$. (Keyes, Des Fleurs pour Algernon, p. 222)

Mais au-delà des références explicites à l'expérience de Robinson Crusoe et de sa solitude, aux textes de Milton, c'est au mythe de Frankenstein que l'ouvrage fait le plus allusion. En effet, Charlie reproche à plusieurs reprises au responsable de l'expérience, le Professeur Nemur, de prétendre qu'il l'a fabriqué, créé. Ainsi, lorsque Charlie se retrouve au Congrès de psychologie à Chicago, où Nemur veut communiquer les résultats de son expérience, il s'écrie :

Cela peut sembler de l'ingratitude, mais c'est l'une des choses qui me déplaisent ici - cette manière de me traiter comme un cobaye. Les rappels constants de Nemur de m'avoir fait ce que je suis ou qu'un jour il y en aura d'autres comme moi qui deviendront vraiment des êtres humains.

Comment puis-je lui faire comprendre qu'il ne m'a pas créé ${ }^{11}$ ? (Keyes, Des Fleurs pour Algernon, p. 120-121)

Il s'agit bien du thème central du roman fantastique de Mary Shelley publié en 1818 Frankenstein or the Modern Prometheus qui raconte comment un savant fou (Victor Frankenstein) crée à partir de cadavres une créature artificielle à qui il insuffle la vie et qui, se révoltant, devient prise de folie meurtrière. Il semblerait ainsi que la lecture de cet ouvrage, considéré comme l'une des premières œuvres de science-fiction, pourrait prolonger et compléter l'étude du roman de Keyes, dans la mesure où tous les deux traitent de la transgression de l'ordre naturel par la science et permettent donc de réfléchir indirectement sur la dimension éthique de la science en général et de la psychologie en particulier. 


\section{BIBLIOGRAPHIE}

GODEFroId Jo, Psychologie, Bruxelles, De Boeck, 2008.

ISANI Shaeda, "The FASP and the Genres within the Genre », dans M. Petit (dir.) et S. Isani (coll.), Aspects de la fiction à substrat professionnel, université Victor Ségalen Bordeaux 2, coll. « Travaux $20.25 », 2004$, p. 25-36.

KEYES Daniel, Flowers for Algernon, [1966], Orlando, Harvest/Harcourt, 2004, 311 p.

KEYES Daniel, Des Fleurs pour Algernon, trad. Georges H. Gallet, Paris, Editions J'ai lu, 1972, 252 p.

LIEURY Alain, Psychologie cognitive, Paris, Dunod, 2004.

PETIT Michel, «Quelques réflexions sur la fiction à substrat professionnel : du général au particulier ", Aspects de la fiction à substrat professionnel, 2004, p. 3-23.

-, « La fiction à substrat professionnel : une autre voie d'accès à l'anglais de spécialité ", Asp, 23/26, 1999, p. 57-81.

RICœUR Paul, Temps et Récit I, Paris, Seuil, 1983, 322 p.

\section{NOTES}

1. Le prix Nebula a été créé par Lloyd Biggle, $\mathrm{J}^{\mathrm{r}}$. Il est décerné une fois par an par les membres de la Science Fiction and Fantasy Writers Academy of America. Le prix Hugo est sans doute le plus prestigieux prix littéraire concernant la science-fiction. Il a été créé en 1953, en hommage à l'auteur Hugo Gernsback (1884-1967), fondateur du premier mensuel de science-fiction, Amazing Stories. Il est décerné, une fois par an, à l'occasion d'une convention mondiale par vote des quelque 5000 membres de la WSFS (World Science Fiction Society).

2. La psychochirurgie est une thérapeutique des troubles mentaux recourant à des interventions chirurgicales sur le cerveau.

3. Ma traduction de «In his definition of the core conventions of the FASP, Petit distinguishes between fiction in which the profession plays a pivotal role (substrat professionnel) and fiction where it is extensively but not indispensably present, for which he suggests the term of adstrat professionnel. » (Isani, p. 26)

4. Ma traduction de «Adstrat: French term borrowed from the field of linguistics to designate a language spoken in a country neighbouring the country of the language of reference, which in the context of our study, would infer the notion of juxtaposition as opposed to source or foundation. » (Isani, p. 26.)

5. When I touched her shoulder she stiffened and trembled, but I pulled her toward me. Then it happened. It started as a hollow buzzing in my ears... an electric saw... far away. Then the cold: arms and legs prickly, and finger numbing. Suddenly, I had the feeling I was being watched. (Keyes, Flowers for Algernon, p. 100.)

6. The solution came to me, just as I was dozing off. Illuminated! Everything fits together, and I see what I should have known from the beginning. No more sleep. I've got to get back to the lab and test this against the results of the computer. This, finally, is the flaw in the experiment. I've found it. (Keyes, Flowers for Algernon, p. 253.) 
7. She hinted at things. She talked around them and expected me to know what she was thinking. And I listened, pretending I understood but inside I was afraid she would see that I missed the point completely. (Keyes, Flowers for Algernon, p. 300.)

8. [En ligne] <http://well.blogs.nytimes.com/...>, consulté le 10 avril 2010.

9. And she said mabey they got no rite to make me smart because if god wantid me to be smart he would have made me born that way. An what about Adem and Eev and the sin with the tree of knowledge and eating the appel and the fall. (Keyes, Flowers for Algernon, p. 16.)

10. I wished I could say that like the House of Atreus or Cadmus we were suffering for the sins of our forefathers, or fulfilling an ancient Greek oracle. (Keyes, Flowers for Algernon, p. 273.)

11. It may sound like ingratitude, but that is one of the things that I resent here - the attitude that I am a guinea pig, Nemur's constant references to having made me what I am, or that someday there will be others like me who will become real humanbeings. How can I make him understand that he did not create me? (Keyes, Flowers for Algernon, p. 145.)

\section{RÉSUMÉS}

Le but de cet article est de tenter de montrer que le best-seller de Daniel Keyes, Flowers for Algernon (1966), appartient au genre de la FASP, en expliquant comment le substrat professionnel ne constitue pas un simple un arrière-plan lexical mais fait partie intégrante du récit.

Après un bref compte rendu d'une expérience d'enseignement de textes littéraires à des adultes spécialisés en psychologie et psychiatrie, qui démontre que l'enseignement de la fiction pouvait être fécond, l'auteur de l'article applique les critères du genre au livre de Keyes, lequel est, en effet, imprégné de questions traitant de psychologie, et qui donc, pourrait être enseigné de façon efficace à des étudiants de psychologie en tant qu'outil pédagogique d'anglais de spécialité.

L'article conclut en soulignant la dimension éthique de l'enseignement de la fiction en langue de spécialité puisque la lecture de la fiction peut susciter des émotions, et plus particulièrement, de l'empathie chez les psychologues en devenir, en développant leurs sentiments altruistes.

This article seeks to demonstrate that Daniel Keyes's best-seller, Flowers for Algernon (1966), belongs to the FASP genre by showing how the professionally-based elements (substrat professionnel) do not constitute a mere lexical background but are an integral part of the narrative.

After a brief account of teaching English classes to adults specializing in psychology and psychiatry through fiction, the author of the paper applies the FASP generic criteria to Keyes's book and analyses the psychology substrat professionnel.

The article concludes by discussing the ethical dimension of the teaching of fiction in ESP related to emotions and empathy generated by the fiction in the psychologists-to-be, thereby developing their altruistic feelings. 
INDEX

Keywords : FASP, Fiction, Psychology, ESP, Flowers for Algernon, substrat, adstrat, narrative, ethics

Mots-clés : FASP, fiction, anglais de spécialité, psychologie, Flowers for Algernon (Des Fleurs pour Algernon), substrat, adstrat, éthique 